\title{
Brazilin prevents against myocardial ischemia-reperfusion injury through the modulation of Nrf2 via the PKC signaling pathway
}

\author{
Bin $\mathrm{Qi}^{1{ }^{1 *}}$, Xiaowen Zhang ${ }^{2 \#}$, Hang $\mathrm{Yu}^{1}$, Yandong Bao ${ }^{1}, \mathrm{Nan} \mathrm{Wu}^{3}$, Dalin Jia ${ }^{1}$ \\ ${ }^{1}$ Department of Cardiology, the First Affiliated Hospital of China Medical University, Shenyang, China; ${ }^{2}$ Medical Research Center, Shengjing Hospital \\ of China Medical University, Shenyang, China; ${ }^{3}$ The Central Laboratory of the First Affiliated Hospital of China Medical University, Shenyang, China \\ Contributions: (I) Conception and design: B Qi, X Zhang, N Wu, D Jia; (II) Administrative support: N Wu, D Jia; (III) Provision of study materials or \\ patients: B Qi, X Zhang, N Wu; (IV) Collection and assembly of data: B Qi, X Zhang, H Yu, Y Bao, N Wu; (V) Data analysis and interpretation: B \\ Qi, X Zhang, H Yu, Y Bao; (VI) Manuscript writing: All authors; (VII) Final approval of manuscript: All authors. \\ "These authors contributed equally to this work. \\ Correspondence to: Prof. Dalin Jia. Department of Cardiology, the First Affiliated Hospital of China Medical University, 155th North of Nanjing \\ Street, Heping, Shenyang 110001, China. Email: jd12001@126.com; Dr. Nan Wu. The Central Laboratory of the First Affiliated Hospital of China \\ Medical University, 155th North of Nanjing Street, Heping District, Shenyang 110001, China. Email: imwunan@163.com.
}

Background: Brazilin, a major ingredient of Caesalpinia sappan L., possesses multiple pharmaceutical activities, although whether or not brazilin exerts any protective effect on myocardial ischemia-reperfusion injury (MIRI) has not yet been reported. The present study determined the cardioprotective effects of brazilin, and elucidated the role of nuclear factor E2-associated factor 2 (Nrf2) in this process.

Methods: Following treatment with brazilin, H9c2 cells were subjected to $6 \mathrm{~h}$ of hypoxia/3 h of reoxygenation. CCK-8 assay and flow cytometry were employed to detect cell viability and apoptosis, respectively. Furthermore, after brazilin treatment, isolated rat hearts underwent $30 \mathrm{~min}$ of ischemia, followed by 90 min of reperfusion. Triphenyltetrazolium chloride (TTC) and terminal deoxynucleotidyl transferasemediated dUTP-biotin nick end labeling (TUNEL) staining were performed to measure myocardial infarct size and apoptosis, respectively. The changes in the levels of proteins were detected by western blotting.

Results: Brazilin treatment dose-dependently led to a significant enhancement in cell viability, a reduction in myocardial infarct size, and a decrease in release of creatine kinase-MB (CK-MB) and lactate dehydrogenase (LDH). Moreover, brazilin also remarkably inhibited apoptosis and led to various improvements in cardiac function. Additionally, brazilin treatment caused a marked alleviation of oxidative stress, as evidenced by the fact that brazilin reduced the accumulation of reactive oxygen species (ROS) and malondialdehyde (MDA), while enhancing the activities of superoxide dismutase (SOD) and glutathione peroxidase (GXH-Px). Mechanistically, it was found that brazilin induced Nrf2 nuclear translocation, with a concomitant upregulation of both heme oxygenase-1 (HO-1) and NAD(P)H quinone oxidoreductase (NQO1) expression. Furthermore, the phosphorylation level and transcriptional activity of Nrf2 were enhanced by brazilin, although these enhancements were abrogated by treatment with a protein kinase $\mathrm{C}$ (PKC) inhibitor. Finally, it was observed that the protective effects of brazilin could be negated through inhibition of Nrf2, which suggested that the cardioprotection afforded by brazilin was Nrf2-dependent.

Conclusions: Taken together, our results have demonstrated that brazilin may afford protection against MIRI through the activation of Nrf2 via the PKC signaling pathway. These results may lay the foundation for the further use of brazilin in the prevention of MIRI in clinical practice.

Keywords: Brazilin; myocardial ischemia-reperfusion injury (MIRI); oxidative stress; nuclear factor E2-associated factor 2 (Nrf2); protein kinase C (PKC)

Submitted Jun 01, 2020. Accepted for publication Nov 15, 2020.

doi: 10.21037/atm-20-4414

View this article at: http://dx.doi.org/10.21037/atm-20-4414 


\section{Introduction}

Acute myocardial infarction (AMI), a major deadly disease worldwide, is commonly caused by acute and persistent coronary artery occlusion (1). Therefore, opening the occlusion of the coronary artery to rapidly restore the blood supply to myocardium is considered to be essential in the treatment of AMI (2). However, certain patients with AMI often experience a deterioration in cardiac function, fatal arrhythmia, and even extended infarct size when the blood supply to myocardium is allowed to recover through implementing percutaneous coronary intervention (PCI) $(3,4)$. This paradoxical phenomenon is described as myocardial ischemia-reperfusion injury (MIRI) (5).

Even though MIRI was discovered more than half a century ago, the specific mechanism has yet to be fully elucidated. Oxidative stress, however, has long been regarded as a pivotal mechanism for MIRI (6). A large amount of reactive oxygen species (ROS) were found to be produced during the process of MIRI (7), and the excessive ROS were shown not only to trigger oxidative stress, but also to destroy cell biological macromolecules, including proteins, lipids and DNA, subsequently leading to myocardial cell death and cardiac function disorder (8). Nuclear factor E2-associated factor 2 (Nrf2) is an important transcription factor that regulates cellular oxidative stress responses, and serves as a key regulator in maintaining intracellular redox homeostasis (9). Nrf2 activity depends upon its dissociation from Kelch-like$\mathrm{ECH}$-associated protein 1 (Keap1), which is regulated by the phosphorylation of Nrf2. For example, Nrf2 phosphorylation via the protein kinase $\mathrm{C}(\mathrm{PKC})$ pathway facilitates the release of Nrf2 from Keap1 (10). Interestingly, recent studies have demonstrated that Nrf2 activation is able to render cardioprotection through coordinating upregulation of both phase II detoxifying enzyme and antioxidant enzyme genes $(11,12)$. Therefore, alleviating oxidative stress injury through modulating Nrf2 activity is potentially a novel strategy for protection against MIRI.

Brazilin, a homoisoflavonoid $\left(\mathrm{C}_{16} \mathrm{H}_{14} \mathrm{O}_{5}\right.$; chemical structure shown in Figure $1 A$ ), is a natural product of Caesalpinia sappan $L$. and it has been widely used as a traditional medicine for a long time in history (13). Brazilin has been reported to possess multiple pharmaceutical effects, including its efficacy in treating depressive and anxiety disorders (14), ameliorating oxidative stress-induced photoaging of skin (15), protecting auditory cells against t-butyl hydroperoxide (t-BHP)-induced cell death $(16,17)$, suppressing high glucose-induced vascular inflammatory processes (18), extending lifespan in C. elegans (19) and so on. These effects are predominantly based on its powerful antioxidant activity, as evidenced by the fact that brazilin has been shown to inhibit antioxidant enzyme gene expression $(16,17)$, lower the accumulation of intracellular ROS $(18,19)$, and increase superoxide dismutase (SOD) activity (19). Interestingly, a recent study reported that brazilin exerted protective effects against renal ischemia-reperfusion injury in rats (20). Taken together, these findings enabled us to hypothesize that brazilin could be used therapeutically to afford protection against MIRI.

In this study, the cardioprotective effects of brazilin on MIRI, and its ability to influence Nrf2 activity, were examined. The results obtained demonstrated that brazilin is able to protect against MIRI both in intro and ex vivo through the activation of Nrf2 via the PKC signaling pathway. The following article is presented in accordance with the ARRIVE reporting checklist (available at http:// dx.doi.org/10.21037/atm-20-4414).

\section{Methods}

Experiments were performed under a project license (No. KT2018009) granted by the institutional/regional/national ethics/committee/ethics board of China Medical University and the Guide for the Care and Use of Laboratory Animals (NIH, USA) were followed closely.

\section{Cell culture and bypoxia/reoxygenation (HR) model}

Rat myocardial cells (the H9c2 cell line) were obtained from the Shanghai Institutes for Biological Sciences (Shanghai, China), and were routinely cultivated in Dulbecco's Modified Eagle Medium (DMEM) supplemented with 10\% fetal bovine serum (FBS) under the conditions of $37^{\circ} \mathrm{C}$ and $5 \% \mathrm{CO}_{2}$.

The HR model was established as described previously (21). Specifically, cells were relocated to an atmosphere of $94 \%$ $\mathrm{N}_{2}, 5 \% \mathrm{CO}_{2}$ and $1 \% \mathrm{O}_{2}$, and subsequently cultured with Earle's medium without glucose and FBS for $6 \mathrm{~h}$ to establish hypoxia. At the end of the incubation, cells were subjected to reoxygenation for $3 \mathrm{~h}$ through cultivating them with normal medium in an atmosphere of $5 \% \mathrm{CO}_{2}$.

\section{Drug treatment in vitro}

Brazilin (CAS no. 474-07-7; purity $\geq 98 \%$ ) and 
A<smiles>Oc1ccc2c(c1)OC[C@]1(O)Cc3cc(O)c(O)cc3[C@H]21</smiles>

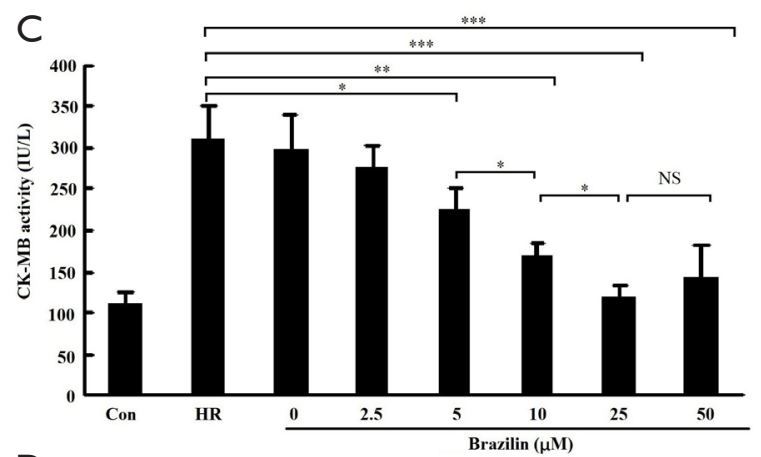

$\mathrm{D}$

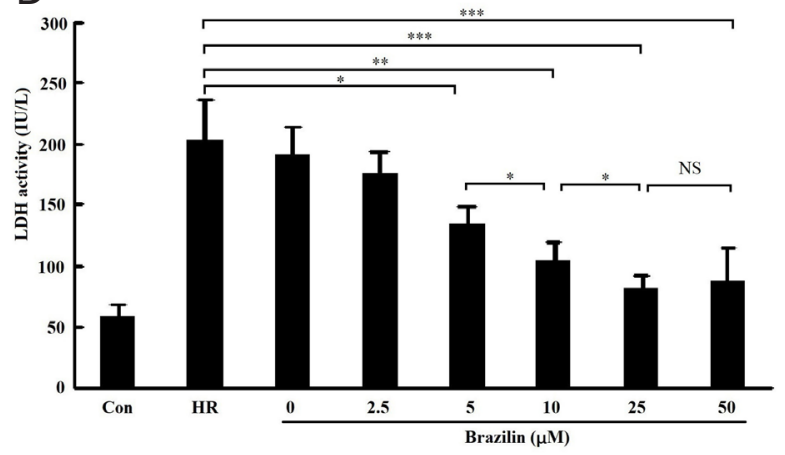

B

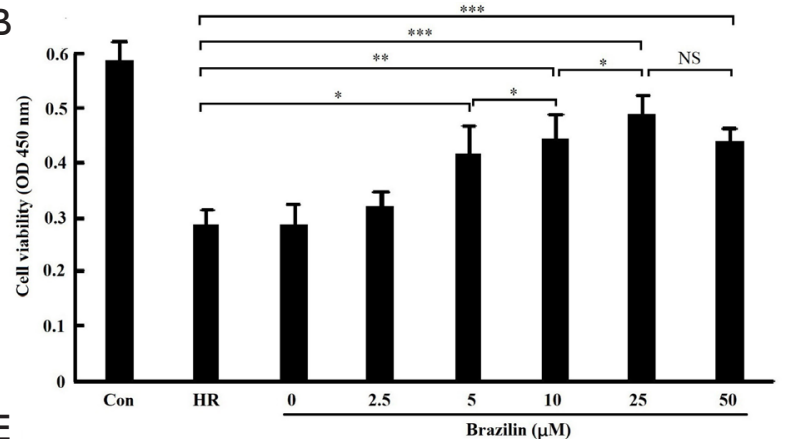

E
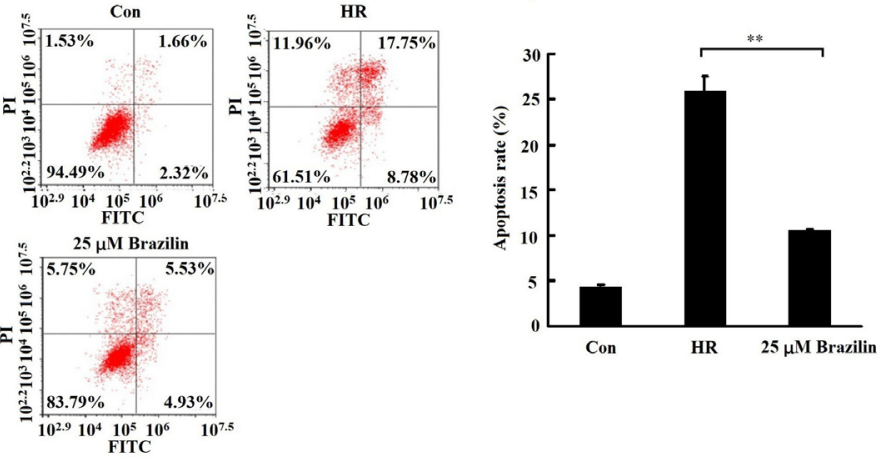

$\mathrm{F}$ Con HR $25 \mu \mathrm{M}$ Brazilin
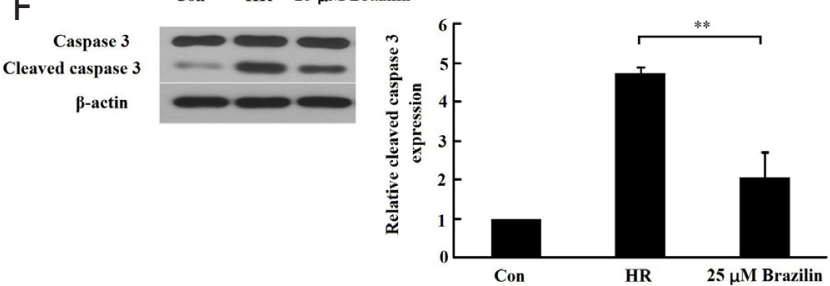

Figure 1 Brazilin protects against myocardial ischemia-reperfusion injury (MIRI) in vitro. Following being treated with various doses of brazilin for $1 \mathrm{~h}$, myocardial cells underwent $6 \mathrm{~h}$ of hypoxia, followed by $3 \mathrm{~h}$ of reoxygenation (HR). (A) Chemical structure of sappanone A. (B) CCK-8 assay. (C) The creatine kinase-MB (CK-MB) activity. (D) Lactate dehydrogenase (LDH) activity. (E) Flow cytometry analysis of cell apoptosis. (F) Western blotting analysis of the protein expression level of caspase-3. All values are expressed as the mean \pm standard deviation (SD) from triplicate independent experiments. *, $\mathrm{P}<0.05 ;{ }^{* *}, \mathrm{P}<0.01$; ***, $\mathrm{P}<0.001$. NS, not significant.

chelerythrine (CAS no. 34316-15-9; purity $\geq 98 \%$ ) were purchased from Wuhan ChemFaces Biochemical Co., Ltd. (Wuhan, China). Brazilin and chelerythrine were dissolved in dimethyl sulphoxide (DMSO) (Sigma-Aldrich; Merck $\mathrm{KGaA}$ ), and further diluted with culture medium until DMSO concentration was $<0.1 \%$. Five different drug concentrations of brazilin $(2.5,5,10,25$ and $50 \mu \mathrm{M})$ were given for $1 \mathrm{~h}$ prior to hypoxia stimulation. Cells were also pretreated with $1 \mu \mathrm{M}$ chelerythrine for $1 \mathrm{~h}$ before brazilin treatment in order to repress PKC activation, as described previously (22).

\section{Cell transfection}

Small interfering RNA targeting Nrf2 (si-Nrf2, 5'-GG GUAAGUCGAGAAGUGUUTTAACACUUCUCGA CUUACCCTT-3') and its negative control (scrambled siRNA, 5 '-UUCUCCGAACGUGUCACGUTTACG UGACACGUUCGGAGAATT-3') were designed and synthesized by GenePharma Co., Ltd. (Shanghai, China). The siRNAs were transfected into cells using Invitrogen ${ }^{\circledR}$ Lipofectamine ${ }^{\circledR} 2000$ (Thermo Fisher Scientific, Inc., Waltham, MA, USA) in accordance with the operation 
guideline. Following transfection for $24 \mathrm{~h}$, cells were further processed for drug treatment, followed by HR.

\section{Cell viability assay}

Cells were cultured in 96-well plates at a concentration of 5,000 cells/well. Subsequently, the cells received treatment with the brazilin and chelerythrine as described above, and underwent HR stimulation. CCK-8 assay (Dojindo Molecular Technologies, Inc., Kumamoto, Japan) was performed to evaluate cell viability following the operation guideline. The absorbance was read at $450 \mathrm{~nm}$ on a microplate reader.

\section{Myocardial enzyme tests}

After the cardiomyocytes had been subjected to the HR treatment, the levels of myocardial enzymes, including lactate dehydrogenase (LDH) and creatine kinase-MB (CK$\mathrm{MB})$, were notably increased. The levels of LDH and CK$\mathrm{MB}$ in the culture medium and coronary effluent were measured using LDH and CK-MB assay kits, respectively (provided by Jiancheng Bioengineering Institute, Nanjing, China), following the operation guideline.

\section{Flow cytometric analysis}

To measure apoptosis in cardiomyocytes, flow cytometry was performed using an annexin $\mathrm{V}$-fluorescein isothiocyanate (FITC)/propidium iodide (PI) kit (Dojindo Molecular Technologies, Inc., Kumamoto, Japan) as previously described (23).

\section{Measurement of ROS content}

The cells were cultured on a tiny glass sheet and fixed with $4 \%$ paraformaldehyde for $15 \mathrm{~min}$. After being washed three times with phosphate buffer saline (PBS), cells were incubated with 2,7-dichlorofluorescin diacetate (DCFHDA), which acts as a sensitive ROS probe (Jiancheng Bioengineering Institute, Nanjing, China), at $37^{\circ} \mathrm{C}$ for $1 \mathrm{~h}$. DCFH-DA-stained cardiomyocytes were observed and photographed under a fluorescence microscope.

\section{Determination of malondialdehyde (MDA), SOD and glutathione peroxidase (GSH-Px) enzymes}

After being digested by trypsin, cells were collected, and subsequently resuspended in $0.5 \mathrm{~mL} \mathrm{PBS}$, prior to being comminuted with an ultrasonic disintegrator. Cell homogenates were centrifugated at $12,000 \mathrm{~g}$ for $10 \mathrm{~min}$, followed by collection of the supernatant. The protein concentration of the supernatant was determined using an enhanced BCA Protein Assay kit (Beyotime Institute of Biotechnology, Haimen, China) following the manufacturer's guidelines. The MDA content, and the activities of SOD and GSH-Px were measured using MDA, SOD and GSH-Px detection kits (all from Jiancheng Bioengineering Institute, Nanjing, China), respectively, following the operation guideline.

\section{Animal experiments}

The animals used in the present study were healthy male Wistar rats (8 weeks, 240-260 g), which were purchased from the Animal Laboratory Center of China Medical University (Shenyang, China). The rats were kept at $22-24{ }^{\circ} \mathrm{C}$ under $12 \mathrm{~h}$ daylight: $12 \mathrm{~h}$ dark conditions, and had free access to clean water. The body weight of the rats was monitored twice a week. The use of animals in the present study was authorized by the Institutional Animal Care and Use Committee of China Medical University, and the Guide for the Care and Use of Laboratory Animals (NIH, USA) were followed closely.

\section{Establishment of the isolated rat heart ischemia- reperfusion model}

Animal anesthesia was implemented through an intraperitoneal injection of sodium pentobarbital $(30 \mathrm{mg} / \mathrm{kg})(23)$. The rat heart was isolated from the thoracic cavity, hung on a Langendorff perfusion device, and perfused with $\mathrm{O}_{2}$-saturated Krebs-Henseleit (K-H) solution at a constant pressure, as described previously (21). Myocardial ischemia-reperfusion was induced by stopping $\mathrm{K}-\mathrm{H}$ solution perfusion for a period of $30 \mathrm{~min}$, followed by reperfusion with $\mathrm{K}-\mathrm{H}$ solution for $90 \mathrm{~min}$. The fluid-filled latex balloon which was connected to a pressure sensor was inserted into the left ventricle to dynamically minitor the alteration of cardiac function using a homodynamic system (MP150; BIOPAC Systems, Inc., Goleta, CA, USA).

\section{Experimental groups}

The animal experiments that were performed consisted of two phases. The concentration gradient of brazilin (12.5, 25 and $50 \mathrm{mg} / \mathrm{kg}$ ) was administrated to determine the most 
effective dose of brazilin against MIRI based on a previous study (24). Forty-eight rats were equally separated into six groups $(n=8)$ for the first phase, as follows: (I) the control group: the isolated heart underwent $120 \mathrm{~min}$ of perfusion without interruption; (II) the ischemia-reperfusion (IR) group: the isolated heart was subjected to interruption of perfusion for $30 \mathrm{~min}$, followed by reperfusion for $90 \mathrm{~min}$; (III) the vehicle group: the rats received an intraperitoneal injection of $1 \mathrm{~mL}$ saline (containing $0.1 \% \mathrm{DMSO}$ ) at $1 \mathrm{~h}$ ahead of heart operation, followed by IR, as described for the IR group; (IV) the $12.5 \mathrm{mg} / \mathrm{kg}$ brazilin treatment group: the rats received an intraperitoneal injection of brazilin $(12.5 \mathrm{mg} / \mathrm{kg}) 1 \mathrm{~h}$ before heart isolation, followed by IR, as described for the IR group; (V) the $25 \mathrm{mg} / \mathrm{kg}$ brazilin treatment group: the rats received $25 \mathrm{mg} / \mathrm{kg}$ brazilin treatment and underwent IR, as described for the $12.5 \mathrm{mg} / \mathrm{kg}$ brazilin group; and (VI) the $50 \mathrm{mg} / \mathrm{kg}$ brazilin treatment group: the rats received $50 \mathrm{mg} / \mathrm{kg}$ brazilin treatment, and underwent IR as described for the $12.5 \mathrm{mg} / \mathrm{kg}$ brazilin group.

To explore the role of $\mathrm{Nrf} 2$ in the cardioprotection of brazilin, 40 rats were equally separated into five groups $(n=8)$ for the second phase, as follows: (I) the control group: the same as described above for the first phase; (II) the IR group: also the same as the first phase; (III) the most effective dose of brazilin treatment group: the rats were administered the most effective dose of brazilin that had been confirmed in the first phase, and then underwent IR as described above for the first phase; (IV) the brazilin + ML385 group: the rats received an intraperitoneal injection of an Nrf2 inhibitor, ML385 (30 mg/kg), 30 min before brazilin administration, based on a previous study (25); and (V) the ML385 group: the rats only received an intraperitoneal injection of ML385 (30 mg/kg), followed by IR, as described above.

\section{Measurement of infarct size}

After the reperfusion finished, the hearts were firstly detached from the device and refrigerated at $-20^{\circ} \mathrm{C}$ for $1 \mathrm{~h}$, then cut into $1 \mathrm{~mm}$-thick sections, finally stained with triphenyltetrazolium chloride (TTC) solution, as described previously (21).

\section{Terminal deoxynucleotidyl transferase-mediated dUTP- biotin nick end labeling (TUNEL) assay}

The heart samples were subjected to formaldehyde fixation and gradient ethanol hydration, before being embedded in wax and made into paraffin sections. TUNEL assay was employed to measure apoptosis in the myocardium using an in situ Cell Death detection kit (Roche Diagnostics, Indianapolis, IN, USA), in accordance with the manufacturer's instructions. The results of TUNEL staining were observed and photographed under a light microscope (Olympus BX51, Japan).

\section{Immunofluorescence staining}

Cells were placed on glass slides, fixed with $4 \%$ paraformaldehyde for $15 \mathrm{~min}$, permeabilized with $0.1 \%$ Triton X-100 for $30 \mathrm{~min}$, and then blocked with goat serum for $15 \mathrm{~min}$ at room temperature. Subsequently, they were incubated with diluted primary antibody against Nrf2 (Abcam, Hong Kong) at $4{ }^{\circ} \mathrm{C}$ overnight, followed by incubation with Cy3-labelled goat anti-mouse $\operatorname{IgG}(1: 2,000$; cat. no. 33208ES60; Yeason, Shanghai, China) for $60 \mathrm{~min}$ at room temperature. Nuclei were re-stained with DAPI, and the results of the staining process were observed under a fluorescence microscope.

\section{Enzyme-linked immunosorbent assay (ELISA)}

The nuclear lysates were extracted from cardiomyocytes using a Nuclear Extraction kit (cat. no. ab113474; Abcam, Cambridge, UK), in accordance with the operation guideline. Nrf2 transcriptional activity was then measured by ELISA using an Nrf2 Transcription Factor Assay kit (cat. no. ab207223; Abcam) in accordance with the operation guideline.

\section{Western blotting}

Total proteins were obtained from the collected cells with RIPA. Nuclear and cytoplasmic proteins were extracted using a Nuclear and Cytoplasmic Protein Extraction kit (Beyotime Institute of Biotechnology, Shanghai, China), and the protein concentration was determined using an enhanced BCA Protein Assay kit (Beyotime Institute of Biotechnology), in accordance with the manufacturer's instructions. Protein samples were thermally denatured, separated by $8 \%$ or $10 \%$ SDS-PAGE electrophoresis, transferred to PVDF membranes and blocked with $1 \%$ bovine serum albumin for $1 \mathrm{~h}$ at room temperature, followed by incubation with diluted primary antibodies, including anti-Nrf2 (cat. no. ab89443), anti-phosphoNrf2 (cat. no. ab76026), anti-caspase 3 (cat. no. ab184787), anti-PKC $\varepsilon$ (cat. no. ab182126), anti-phospho-PKC $\varepsilon$ (cat. 
no. ab108972), anti-heme oxygenase-1 (anti-HO-1) (cat. no. ab68477) and anti-NAD(P)H quinone oxidoreductase (anti-NQO1) (cat. no. ab28947) (all antibodies diluted 1:1,000 and purchased from Abcam) at $4{ }^{\circ} \mathrm{C}$ overnight. After being washed three times with PBS, the membranes were incubated with horseradish peroxidase (HRP)-labelled IgG (1:5,000; Zhongshan Jinqiao Biotechnology, Beijing, China) at room temperature for $30 \mathrm{~min}$. The membranes were then developed using a BeyoECL Plus kit (Beyotime Institute of Biotechnology) following the operation guideline. Image $\mathrm{J} 2 \mathrm{x}$ analysis software (NIH, Bethesda, MD, USA) was employed to analyze relative densitometry values.

\section{Statistical analysis}

All values are presented as the mean \pm standard deviation (SD). The statistical significance between groups were determined using one-way analysis of variance, followed by the least significant difference post-hoc test. SPSS version 17.0 software (SPSS Inc., Chicago, IL, USA) was employed to conduct all statistical analyses, and $\mathrm{P}<0.05$ was considered statistically significant.

\section{Results}

\section{Brazilin protected against MIRI in vitro}

First, the effects of various concentrations of brazilin (0$50 \mu \mathrm{M})$ on cell viability and the release of cardiac enzymes in the HR-induced cardiomyocytes were examined. The results showed that concentrations of brazilin ranging from 5 to $25 \mu \mathrm{M}$ dose-dependently led to a notable enhancement in cell viability and a reduction in the release of $\mathrm{CK}$ $\mathrm{MB}$ and LDH. However, no further enhancements in the effectiveness of brazilin were observed in the higher concentration of brazilin $(50 \mu \mathrm{M})$ treatment group (Figure 1B,C,D). Accordingly, $25 \mu \mathrm{M}$ brazilin was chosen as the concentration for further investigations in vitro. Moreover, the cell apoptotic rate (Figure 1E) and expression level of cleaved caspase 3 (Figure $1 F$ ) were shown to be repressed by brazilin treatment. Altogether, these findings suggested that brazilin could protect against MIRI in vitro.

\section{Brazilin ameliorated MIRI ex vivo}

Subsequently, the cardioprotective effects of brazilin in an isolated rat heart model were examined. As shown in Figure $2 A$, the myocardial infarct size was dose-dependently reduced by brazilin, ranging from 12.5 to $25 \mathrm{mg} / \mathrm{kg}$. Furthermore, similar results were also obtained with respect to the $\mathrm{CK}-\mathrm{MB}$ and $\mathrm{LDH}$ (Figure 2B,C). However, no additional enhancement of the effects were shown in the higher dose of brazilin $(50 \mathrm{mg} / \mathrm{kg})$ treatment group. Therefore, $25 \mathrm{mg} / \mathrm{kg}$ brazilin was selected as the dose for further experiments ex vivo. Moreover, the percentage of apoptotic cells was decreased by brazilin treatment (Figure 2D). In addition, even though no significant changes were observed in terms of heart rate (Figure $2 E$ ), the higher values of LVDP and $\pm \mathrm{dp} / \mathrm{dt}$ were preserved in the brazilin treatment group compared with the IR group (Figure $2 F, G, H)$. Altogether, these findings revealed that brazilin could ameliorate MIRI ex vivo.

\section{Brazilin alleviated HR-induced oxidative stress}

To investigate the effects of brazilin on HR-induced oxidative stress, the levels of ROS and MDA and the activities of the anti-oxidative enzymes SOD and GXHPx following brazilin treatment were both examined. The results showed that brazilin decreased the accumulation of ROS and MDA in HR-induced cardiomyocytes (Figure $3 A, B)$. Brazilin was also found to enhance the activities of SOD and GXH-Px (Figure 3C,D). Altogether, these results suggested that brazilin could alleviate HRinduced oxidative stress.

\section{Brazilin modulated the activity of Nvf2 via the PKC patbway}

To elucidate how the levels of oxidative stress may be regulated by brazilin, the effect of brazilin on the regulation of Nrf2, a key transcription factor to oxidative stress, was subsequently assessed. The results indicated that brazilin could induce Nrf2 nuclear translocation, as evidenced by a notable increase in the expression level of Nrf2 in the nuclei, accompanied by a marked decrease in the expression of $\mathrm{Nrf} 2$ in the cytoplasm (Figure $4 A, B$ ). Additionally, HO-1 and NQO1, two main downstream target genes of Nrf2, were downregulated following brazilin treatment (Figure $4 C, D$ ). Altogether, these findings revealed that brazilin could stimulate the transcriptional activity of Nrf2.

Since Nrf2 nuclear translocation may be regulated by PKC-mediated phosphorylation (26), the influence of brazilin on the phosphorylation of PKCE and Nrf2 was subsequently examined. The results revealed that the phosphorylation level of PKCe (Figure 5A) and Nrf2 (Figure 
A
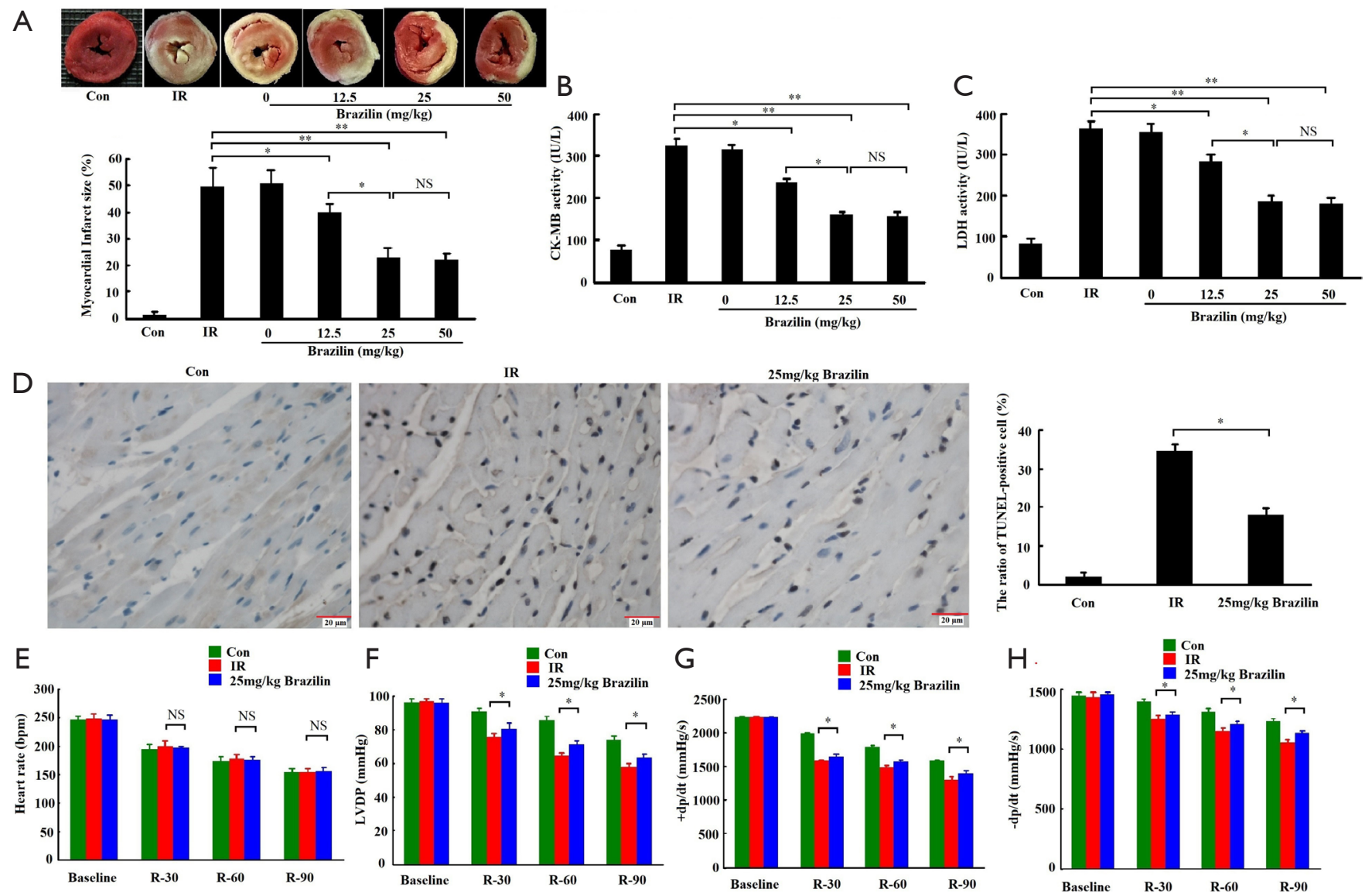

Figure 2 Brazilin ameliorates myocardial ischemia-reperfusion injury (MIRI) ex vivo. Wistar rats received an intraperitoneal injection of different doses of brazilin $1 \mathrm{~h}$ before heart isolation, and then the isolated hearts were subjected to $30 \mathrm{~min}$ of ischemia, followed by $90 \mathrm{~min}$ of reperfusion. (A) Triphenyltetrazolium chloride (TTC) staining. (B) The creatine kinase-MB (CK-MB) activity in coronary effluent. (C) Lactate dehydrogenase (LDH) activity in coronary effluent. (D) Myocardial apoptosis was measured by terminal deoxynucleotidyl transferase-mediated dUTP-biotin nick end labeling (TUNEL) staining (magnification, $\times 400)(n=6)$. The nuclei in apoptotic cells presented brown, and normal nuclei presented blue. Scale bar $=20 \mu \mathrm{m}$. Changes in cardiac function parameters were collected $1 \mathrm{~min}$ prior to ischemia (baseline), after 30 (R-30), 60 (R-60) and 90 (R-90) min of reperfusion. (E) Heart rate. (F) Left ventricular developed pressure (LVDP). (G) Positive first-order derivative of ventricular pressure $(+\mathrm{dp} / \mathrm{dt})$. (H) Negative first-order derivative of ventricular pressure (-dp/dt). All values are expressed as the mean \pm standard deviation $(\mathrm{SD})(\mathrm{n}=8)$. *, $\mathrm{P}<0.05$; **, $\mathrm{P}<0.01$. NS, not significant.

$5 B$ ), as well as the transcriptional activity of Nrf2 (Figure $5 C$ ), were increased by brazilin. However, the reinforced phosphorylation level and transcriptional activity of $\mathrm{Nrf} 2$ by brazilin were found to be abrogated by a PKC inhibitor (Figure 5B,C). Therefore, these results further suggested that brazilin was activating Nrf2 through the PKC pathway.

\section{The cardioprotection of brazilin might be rescued via inbibition of $\mathrm{N} v f 2$}

To confirm the role of $\mathrm{Nrf} 2$ in the cardioprotection of brazilin, Nrf2 was inhibited by si-Nrf2 in vitro or via an Nrf2 inhibitor (ML385) in vivo, followed by brazilin treatment. The enhancement in cell viability and reduction in the rate of release of CK-MB and LDH mediated by brazilin were effectively rescued by treatment with si-Nrf2 in vitro (Figure $6 A, B, C$ ). Similarly, the beneficial effects of brazilin on myocardial necrosis were also abrogated by treatment with ML385 (Figure 6D,E,F). These results suggested that the protective effects of brazilin on MIRI were abolished via the inhibition of $\mathrm{Nrf} 2$, and confirmed that brazilin exerted its protection against MIRI via a 
A
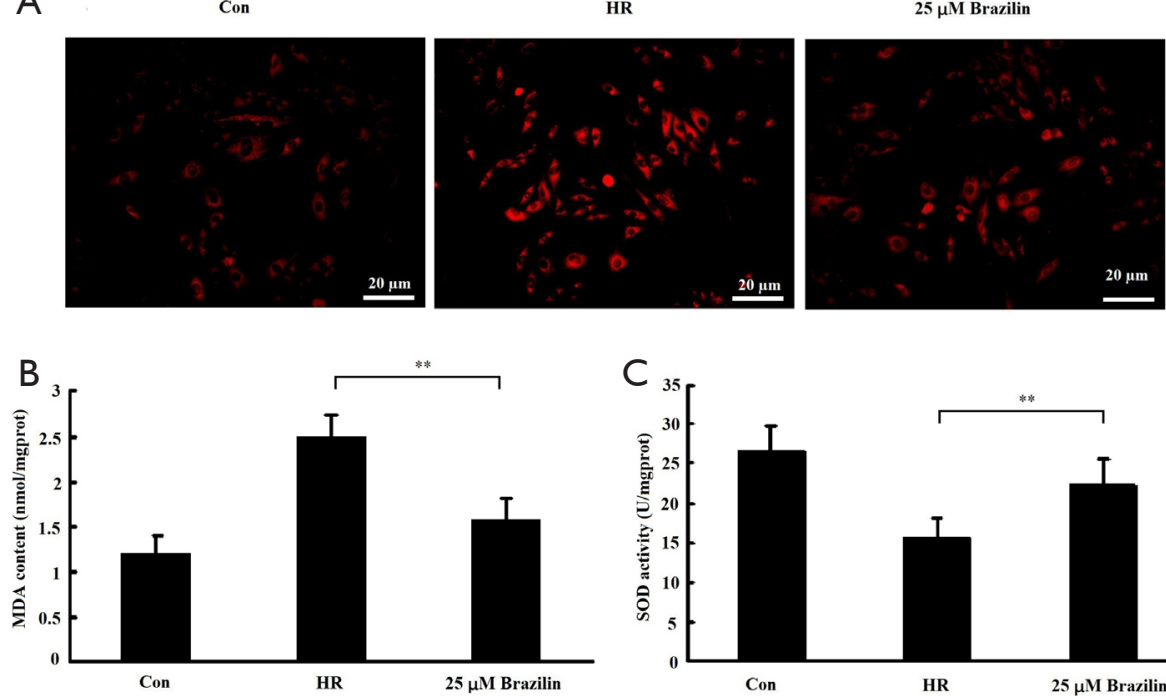
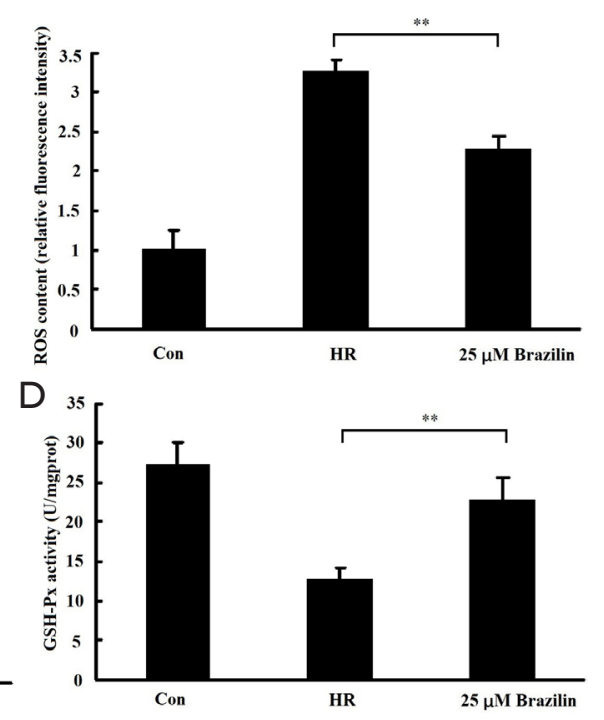

Figure 3 Brazilin alleviates hypoxia/reoxygenation (HR)-induced oxidative stress. Following being treated with $25 \mu M$ brazilin for 1 h, myocardial cells underwent $6 \mathrm{~h}$ of hypoxia, followed by $3 \mathrm{~h}$ of reoxygenation (HR). (A) Analysis of reactive oxygen species (ROS) levels in cells using a DCFH-DA probe (immunofluorescence staining). Scale bar $=20 \mu \mathrm{m}$. The ROS level was calculated by the mean fluorescence intensity. (B) Malondialdehyde (MDA) content. (C) Superoxide dismutase (SOD) activity. (D) Glutathione peroxidase (GSH-Px) activity. All values are presented as the mean \pm standard deviation $(\mathrm{SD})(\mathrm{n}=6) .{ }^{* *}, \mathrm{P}<0.01$.

mechanism involving Nrf2.

\section{Discussion}

Caesalpinia sappan L., a type of flowering tree belonging to the legume family, is mainly distributed in certain Asian countries, including India, Myanmar and Vietnam. Its dried heartwood, usually used as a fork medicine, is used to treat ulcers, diarrhea, epilepsy, traumatic disease and menstrual disorders in those countries (27). A body of experimental evidence has demonstrated that the extract of Caesalpinia sappan L. possesses anti-oxidative (16), anti-inflammatory (28) and antimicrobial activities (29). In addition, sappanone $\mathrm{A}$, another active component of Caesalpinia sappan L. that is similar to brazilin, has been demonstrated to protect against MIRI both in vitro and ex vivo $(30,31)$. However, whether brazilin is able to exert any protective effects on MIRI has not yet been reported. In this study, it was shown that brazilin could alleviate MIRI in a dose-dependent manner in vitro and ex vivo. To the best of the authors' knowledge, this is the first study to have been published on the role of cardioprotection afforded by brazilin, and these findings will lay the foundation for the further use of brazilin in terms of the prevention of MIRI in clinical practice.
Previous studies have shown that the majority of the beneficial pharmaceutical effects exerted by brazilin are concerned with its regulation on oxidative stress (14-19). For example, Wang et al. (14) suggested that brazilin exerted antidepressant- and anxiolytic-like effects in mice with chronically mild stress (CMS)-induced depression, probably through inhibiting oxidative stress. Moreover, Jayakumar et al. (18) suggested that brazilin could suppress high glucose-induced vascular inflammatory processes partly via inhibiting ROS production. In agreement with previous findings, it was also shown in the present study that brazilin could inhibit the accumulation of ROS and MDA in HR-induced cardiomyocytes, while the activities of antioxidant enzymes in the cardiomyocytes were stimulated. These results suggested that the anti-MIRI effect of brazilin is based, at least in part, on its coordination of the cellular antioxidant defenses and maintenance of the redox balance under conditions of MIRI.

Mounting evidence has shown that Nrf2 exerts a critical role in MIRI $(11,12)$. A significant increase in myocardial infarct size was demonstrated in Nrf2-knockout mice that were subjected to IR (32). On the other hand, Nrf2 could be activated by ischemic preconditioning and ischemic postconditioning, two well-established endogenous cardioprotective mechanisms, subsequently 

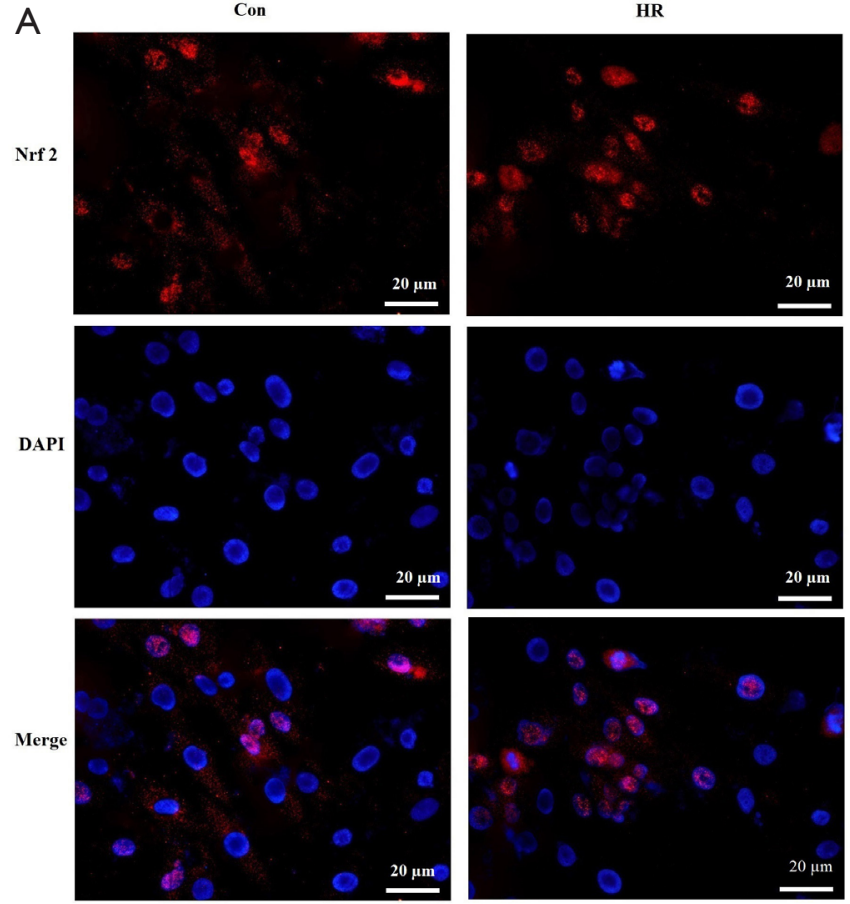

c
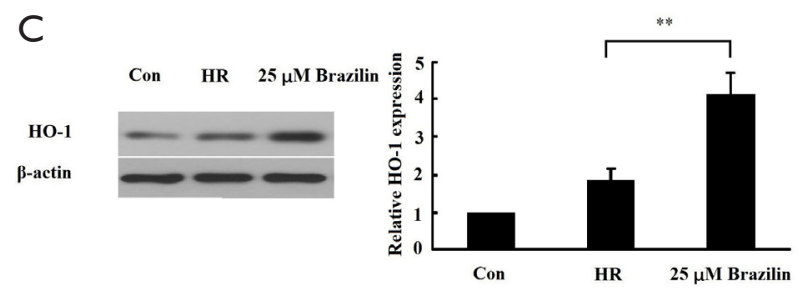

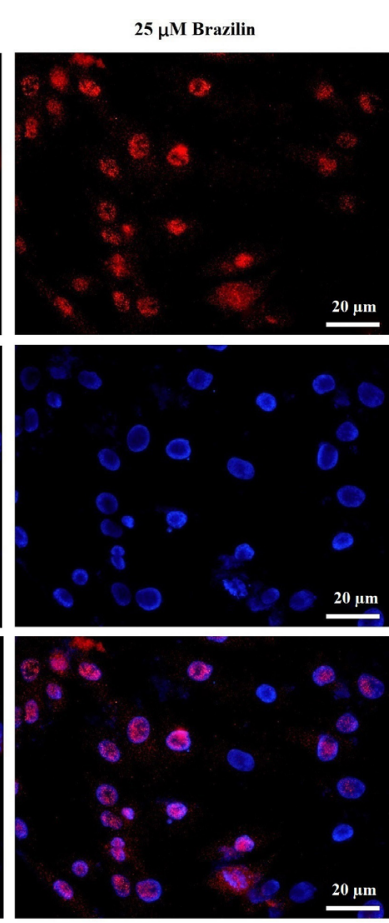

$\mathrm{D}$

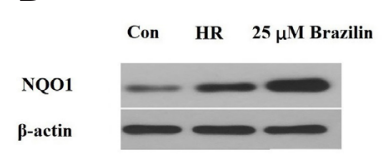

B
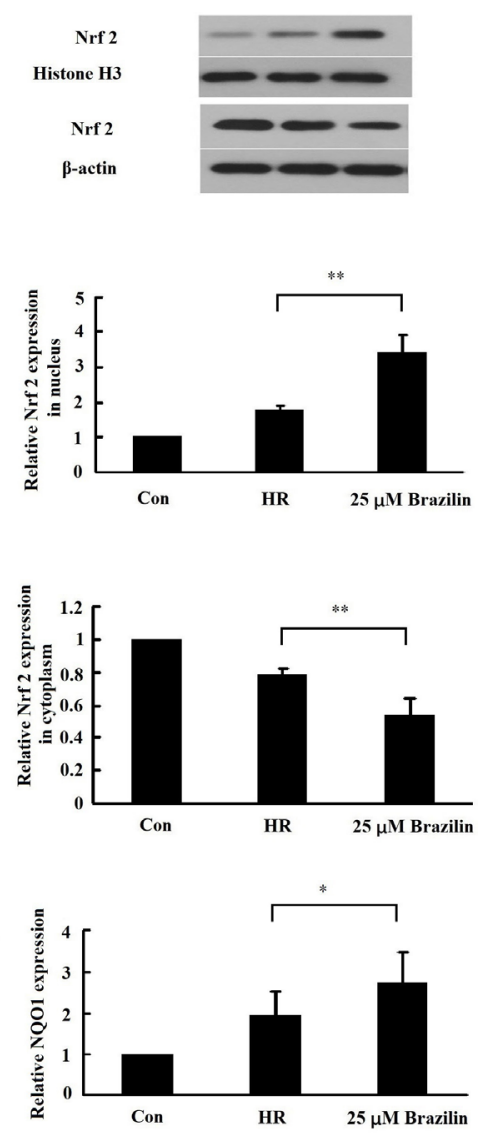

Figure 4 Brazilin promotes Nrf2 nuclear translocation and upregulates the expression of its target genes. Following being treated with various doses of brazilin for $1 \mathrm{~h}$, myocardial cells underwent $6 \mathrm{~h}$ of hypoxia, followed by $3 \mathrm{~h}$ of reoxygenation (HR). (A) Nrf2 nuclear translocation was detected via immunofluorescence staining. Scale bar $=20 \mu \mathrm{m}$. (B) Western blotting analysis of the expression level of Nrf2 in the cytoplasm and nuclei. (C) Western blotting analysis of heme oxygenase-1 (HO-1) expression. (D) Western blotting analysis of $\mathrm{NAD}(\mathrm{P}) \mathrm{H}$ quinone dehydrogenase 1 (NQO1) expression. All values are expressed as the mean \pm standard deviation $(\mathrm{SD})(\mathrm{n}=3)$. *, $\mathrm{P}<0.05 ;$ **, $\mathrm{P}<0.01$.

inducing antioxidant enzymes to protect the heart against MIRI $(33,34)$. In this study, it was shown that Nrf2 was translocated into the nucleus, subsequently leading to an increase in HO-1 and NQO1 expression following brazilin treatment, findings that were consistent with those of Choi et al. (17), who were working with t-BHP-induced House Ear Institute-Organ of Corti 1 (HEI-OC1) cells. Additionally, the present study revealed that the protective effect of brazilin was abrogated by inhibition of $\mathrm{Nrf2}$ with si-Nrf2 and an Nrf2 inhibitor, which further suggested that the cardioprotective effects of brazilin were Nrf2dependent. Taken together, it was possible to conclude that brazilin protected against MIRI via activation of $\mathrm{Nrf2}$.
It has been reported that the activation of $\mathrm{Nrf2}$ induced by oxidative stress is involved with PKC and the phosphoinositide 3-kinase (PI3K)/Akt and extracellularsignal-regulated kinase (ERK) pathways 12). Choi et al. (17) reported that an increase in the expression of HO-1 induced by brazilin was dependent on activation of the PI3K/Akt and ERK pathways. However, whether PKC is involved with the activation of $\mathrm{Nrf} 2$ induced by brazilin has yet to be determined. Our present study showed that brazilin could induce PKC activation by enhancing its phosphorylation. An accumulating body of evidence has shown that PKC activation regulates Nrf2 transcription. For example, direct phosphorylation of serine- 40 of $\mathrm{Nrf2}$ by PKC led to the 

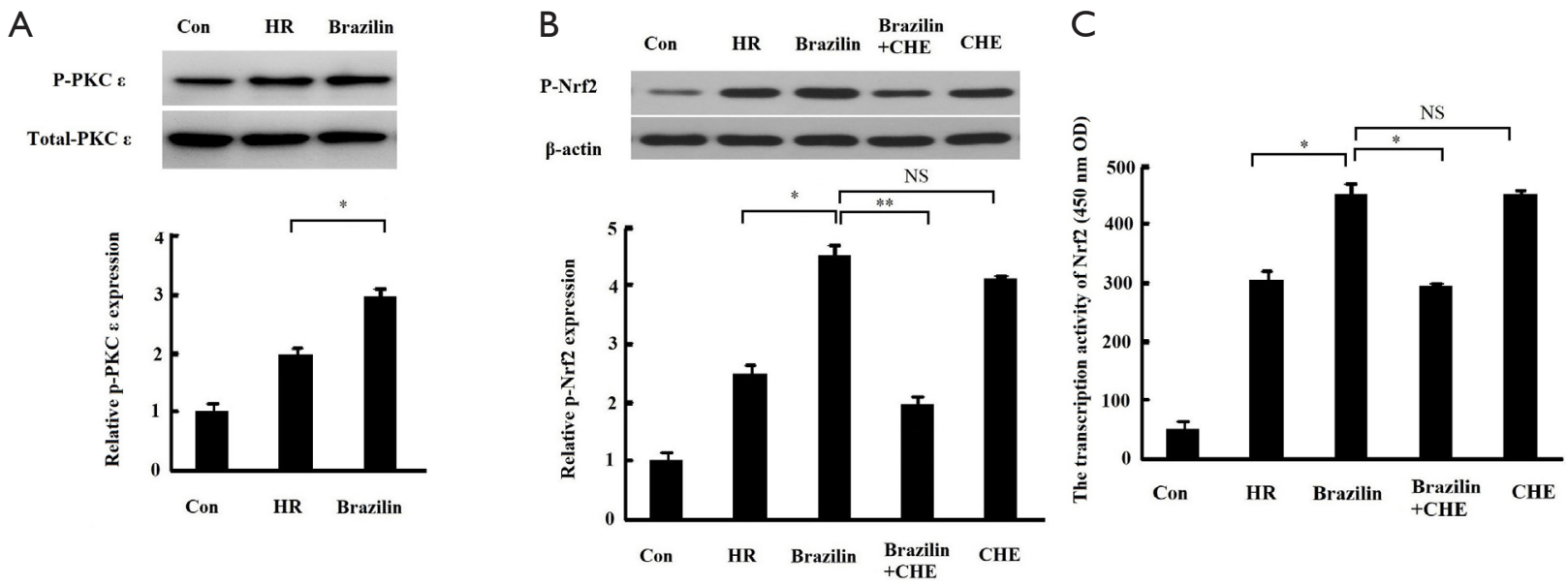

Figure 5 Brazilin activates Nrf2 through the PKC pathway. Following being treated with various doses of brazilin for 1 h, myocardial cells underwent $6 \mathrm{~h}$ of hypoxia, followed by $3 \mathrm{~h}$ of reoxygenation (HR). Cells were treated with $1 \mu \mathrm{M}$ chelerythrine (CHE), a PKC inhibitor, $1 \mathrm{~h}$ before brazilin treatment. (A) Western blotting analysis of the phosphorylation of PKCe. (B) Western blotting analysis of the phosphorylation of Nrf2 (P-Nrf2). (C) The transcriptional activity of Nrf2 was measured by enzyme-linked immunosorbent assay (ELISA). All values are expressed as the mean \pm standard deviation $(\mathrm{SD}),(\mathrm{n}=3) .{ }^{*}, \mathrm{P}<0.05 ;{ }^{* *}, \mathrm{P}<0.01$. NS, not significant.
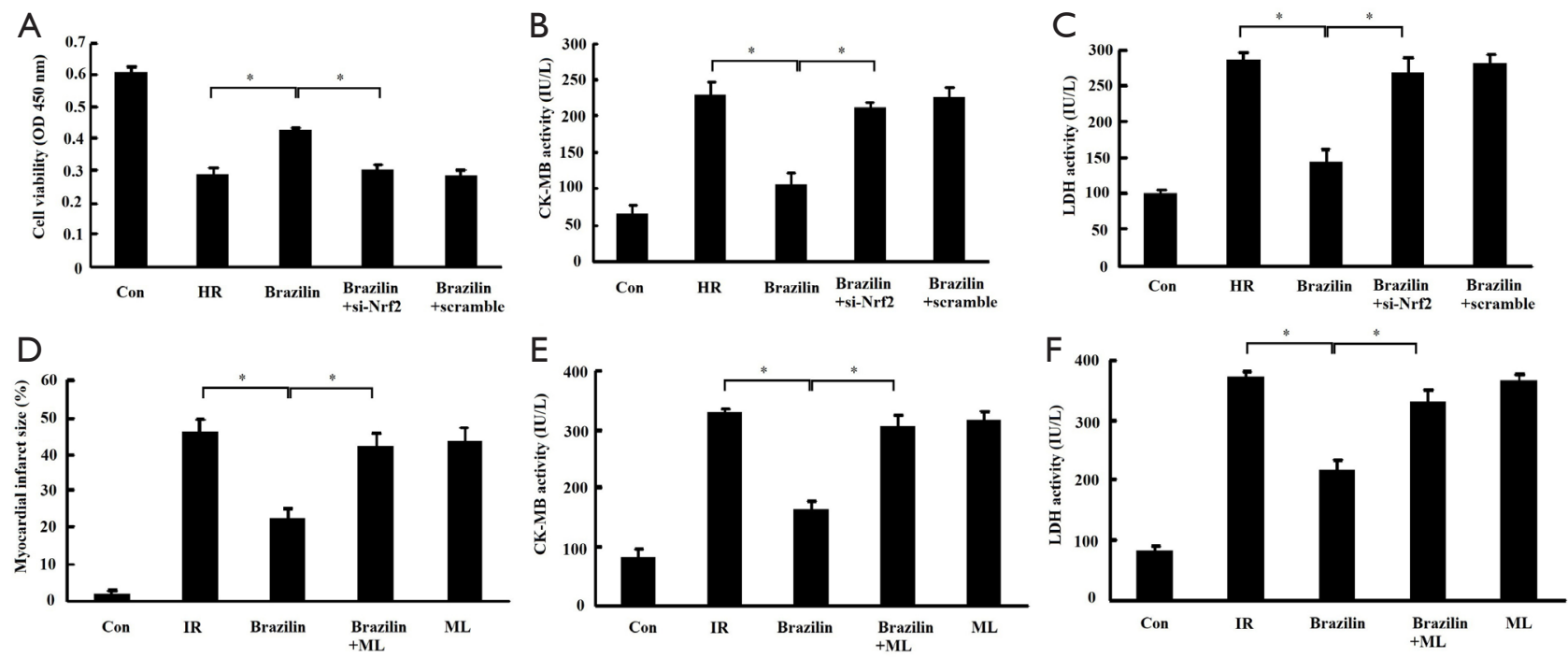

Figure 6 The cardioprotection of brazilin was rescued by inhibition of Nrf2. H9c2 cells were transfected with small interfering RNA targeting Nrf2 (si-Nrf2) and its negative control (scrambled siRNA). Following transfection for 24 h, cells were further processed for brazilin treatment, then subjected to $6 \mathrm{~h}$ of hypoxia, followed by $3 \mathrm{~h}$ of reoxygenation (HR). (A) CCK- 8 assay. (B) The creatine kinase-MB (CK-MB) activity in the culture medium. (C) Lactate dehydrogenase (LDH) activity in the culture medium. All values are expressed as the mean \pm standard deviation (SD) from triplicate independent experiments. * $\mathrm{P}<0.05$. Following being intraperitoneally administered with brazilin $(25 \mathrm{mg} / \mathrm{kg}$ ) for $1 \mathrm{~h}$, the hearts were isolated and subjected to 30-min ischemia, followed by 90 -min reperfusion. ML385 (30 mg/kg), an Nrf2 inhibitor, was intraperitoneally given for $30 \mathrm{~min}$ before brazilin treatment. (D) Triphenyltetrazolium chloride (TTC) staining. (E) Creatine kinase-MB (CK-MB) activity in the coronary effluent. (F) Lactate dehydrogenase (LDH) activity in the coronary effluent. All values are presented as the mean $\pm \mathrm{SD}(\mathrm{n}=8)$. *, $\mathrm{P}<0.05$. 
release of Nrf2 from Keap1, a physiological suppressor of Nrf2, thereby promoting translocation of $\mathrm{Nrf} 2$ to the nucleus $(35,36)$. The results of the present study have corroborated that both the phosphorylation and the transcriptional activity of $\mathrm{Nrf2}$ were reinforced by brazilin treatment, but abolished by PKC inhibitors, suggesting that the activation of $\mathrm{Nrf2}$ by brazilin is mediated via the PKC pathway. These findings provide novel information in terms of understanding how the induction of $\mathrm{Nrf} 2$ by brazilin occurs.

There were certain limitations associated with the present study. Since the isolated heart lacks its usual neural and humoral regulation, the MIRI model established by the Langendorff method is not entirely reflective of the pathophysiological alterations during MIRI. The cardioprotective mechanism of brazilin should also be examined further in vivo.

In conclusion, the present results have demonstrated that brazilin may protect against MIRI via activation of Nrf2 through the PKC pathway. These results may lay the foundation for the further use of brazilin in the prevention of MIRI in clinical practice.

\section{Acknowledgments}

Funding: This work was supported by the National Natural Science Foundation of China (grant nos. 81800232, 81802698 and 81670320).

\section{Footnote}

Reporting Checklist: The authors have completed the ARRIVE reporting checklist. Available at http://dx.doi. org/10.21037/atm-20-4414

Data Sharing Statement: Available at http://dx.doi. org/10.21037/atm-20-4414

Conflicts of Interest: All authors have completed the ICMJE uniform disclosure form (available at http://dx.doi. org/10.21037/atm-20-4414). The authors have no conflicts of interest to declare.

Ethical Statement: The authors are accountable for all aspects of the work in ensuring that questions related to the accuracy or integrity of any part of the work are appropriately investigated and resolved. Experiments were performed under a project license (No. KT2018009) granted by the institutional/regional/national ethics/ committee/ethics board of China Medical University and the Guide for the Care and Use of Laboratory Animals (NIH, USA) were followed closely.

Open Access Statement: This is an Open Access article distributed in accordance with the Creative Commons Attribution-NonCommercial-NoDerivs 4.0 International License (CC BY-NC-ND 4.0), which permits the noncommercial replication and distribution of the article with the strict proviso that no changes or edits are made and the original work is properly cited (including links to both the formal publication through the relevant DOI and the license). See: https://creativecommons.org/licenses/by-nc-nd/4.0/.

\section{References}

1. Benjamin EJ, Virani SS, Callaway CW, et al. Heart disease and stroke statistics-2018 update: a report from the American Heart Association. Circulation 2018;137:e67-492.

2. Gibson CM, Pride YB, Frederick PD, et al. Trends in reperfusion strategies, door-to-needle and door-to-balloon times, and in-hospital mortality among patients with STsegment elevation myocardial infarction enrolled in the National Registry of Myocardial Infarction from 1990 to 2006. Am Heart J 2008;156:1035-44.

3. Liu $M$, Zhang $P$, Chen $M$, et al. Aging might increase myocardial ischemia/reperfusion-induced apoptosis in humans and rats. Age (Dordr) 2012;34:621-32.

4. Reinstadler SJ, Stiermaier T, Eitel C, et al. Relationship between diabetes and ischaemic injury among patients with revascularized ST-elevation myocardial infarction. Diabetes Obes Metab 2017;19:1706-13.

5. Hausenloy DJ, Yellon DM. Myocardial ischemiareperfusion injury: a neglected therapeutic target. J Clin Invest 2013;123:92-100.

6. Yellon DM, Hausenloy DJ. Myocardial reperfusion injury. N Engl J Med 2007;357:1121-35.

7. Cadenas S. ROS and redox signaling in myocardial ischemia-reperfusion injury and cardioprotection. Free Radic Biol Med 2018;117:76-89.

8. Redza-Dutordoir M, Averill-Bates DA. Activation of apoptosis signalling pathways by reactive oxygen species. Biochim Biophys Acta 2016;1863:2977-92.

9. Kasai S, Shimizu S, Tatara Y, et al. Regulation of Nrf2 by mitochondrial reactive oxygen species in physiology and pathology. Biomolecules 2020;10:320. 
10. Bloom DA, Jaiswal AK. Phosphorylation of Nrf2 at Ser40 by protein kinase $\mathrm{C}$ in response to antioxidants leads to the release of $\mathrm{Nrf2}$ from INrf2, but is not required for Nrf2 stabilization/accumulation in the nucleus and transcriptional activation of antioxidant response elementmediated NAD(P)H:quinone oxidoreductase-1 gene expression. J Biol Chem 2003;278:44675-82.

11. Chen QM, Maltagliati AJ. Nrf2 at the heart of oxidative stress and cardiac protection. Physiol Genomics 2018;50:77-97.

12. Shen $\mathrm{Y}$, Liu X, Shi J, et al. Involvement of Nrf2 in myocardial ischemia and reperfusion injury. Int $\mathrm{J}$ Biol Macromol 2019;125:496-502.

13. Nirmal NP, Rajput MS, Prasad RG, et al. Brazilin from Caesalpinia sappan heartwood and its pharmacological activities: a review. Asian Pac J Trop Med 2015;8:421-30.

14. Wang X, Xiu Z, Du Y, et al. Brazilin treatment produces antidepressant- and anxiolytic-like effects in mice. Biol Pharm Bull 2019;42:1268-74.

15. Hwang HS, Shim JH. Brazilin and Caesalpinia sappan L. extract protect epidermal keratinocytes from oxidative stress by inducing the expression of GPX7. Chin J Nat Med 2018;16:203-9.

16. Choi BM, Lee JA, Gao SS, et al. Brazilin and the extract from Caesalpinia sappan L. protect oxidative injury through the expression of heme oxygenase-1. Biofactors 2007;30:149-57.

17. Choi BM, Kim BR. Upregulation of heme oxygenase-1 by brazilin via the phosphatidylinositol 3-kinase/Akt and ERK pathways and its protective effect against oxidative injury. Eur J Pharmacol 2008;580:12-8.

18. Jayakumar T, Chang CC, Lin SL, et al. Brazilin ameliorates high glucose-induced vascular inflammation via inhibiting ROS and CAMs production in human umbilical vein endothelial cells. Biomed Res Int 2014;2014:403703.

19. Lee EB, Xing MM, Kim DK. Lifespan-extending and stress resistance properties of brazilin from Caesalpinia sappan in Caenorhabditis elegans. Arch Pharm Res 2017;40:825-35.

20. Jia Y, Zhao J, Liu M, et al. Brazilin exerts protective effects against renal ischemia-reperfusion injury by inhibiting the NF- $\kappa \mathrm{B}$ signaling pathway. Int J Mol Med 2016;38:210-6.

21. Wu N, Zhang X, Bao Y, et al. Down-regulation of GAS5 ameliorates myocardial ischaemia/reperfusion injury via the miR-335/ROCK1/AKT/GSK-3 $\beta$ axis. J Cell Mol Med 2019;23:8420-31.

22. Kuo HF, Lai YJ, Wu JC, et al. A xanthine-derivative $\mathrm{K}(+)$-channel opener protects against serotonin-induced cardiomyocyte hypertrophy via the modulation of protein kinases. Int J Biol Sci 2013;10:64-72.

23. Zhao Y, Guo Y, Chen Y, et al. Curculigoside attenuates myocardial ischemia-reperfusion injury by inhibiting the opening of the mitochondrial permeability transition pore. Int J Mol Med 2020;45:1514-24.

24. Jia Y, Li Y, Song Y, et al. A simple high-performance liquid chromatographic method for the determination of brazilin and its application to a pharmacokinetic study in rats. J Ethnopharmacol 2014;151:108-13.

25. Sun J, Yu X, Huangpu H, et al. Ginsenoside Rb3 protects cardiomyocytes against hypoxia/reoxygenation injury via activating the antioxidation signaling pathway of PERK/ Nrf2/HMOX1. Biomed Pharmacother 2019;109:254-61.

26. Niture SK, Kaspar JW, Shen J, et al. Nrf2 signaling and cell survival. Toxicol Appl Pharmacol 2010;244:37-42.

27. Yan Y, Chen YC, Lin YH, et al. Brazilin isolated from the heartwood of Caesalpinia sappan L induces endotheliumdependent and -independent relaxation of rat aortic rings. Acta Pharmacol Sin 2015;36:1318-26.

28. Tewtrakul S, Tungcharoen P, Sudsai T, et al. Antiinflammatory and wound healing effects of Caesalpinia sappan L. Phytother Res 2015;29:850-6.

29. Zuo GY, Han ZQ, Han J, et al. Antimicrobial activity and synergy of antibiotics with two biphenyl compounds, protosappanins A and B from Sappan Lignum against methicillin-resistant Staphylococcus aureus strains. J Pharm Pharmacol 2015;67:1439-47.

30. Shi X, Tao G, Ji L, et al. Sappanone A alleviates hypoxia/reoxygenation-induced cardiomyocytes injury through inhibition of mitochondrial apoptosis and activation of PI3K-Akt-Gsk-3 $\beta$ pathway. Biosci Rep 2020;40:BSR20192442.

31. Shi X, Tao G, Ji L, et al. Sappanone A Protects Against Myocardial Ischemia Reperfusion Injury by Modulation of Nrf2. Drug Des Devel Ther 2020;14:61-71.

32. Xu B, Zhang J, Strom J, et al. Myocardial ischemic reperfusion induces de novo $\mathrm{Nrf2}$ protein translation. Biochim Biophys Acta 2014;1842:1638-47.

33. Zhang X, Xiao Z, Yao J, et al. Participation of protein kinase $\mathrm{C}$ in the activation of $\mathrm{Nrf} 2$ signaling by ischemic preconditioning in the isolated rabbit heart. Mol Cell Biochem 2013;372:169-79.

34. Buelna-Chontal M, Guevara-Chávez JG, Silva-Palacios A, et al. Nrf2-regulated antioxidant response is activated by protein kinase $\mathrm{C}$ in postconditioned rat hearts. Free Radic Biol Med 2014;74:145-56.

35. Huang HC, Nguyen T, Pickett CB. Phosphorylation of 
$\mathrm{Nrf2}$ at Ser-40 by protein kinase $\mathrm{C}$ regulates antioxidant response element-mediated transcription. J Biol Chem 2002;277:42769-74.

Cite this article as: Qi B, Zhang $\mathrm{X}$, Yu H, Bao Y, Wu N, Jia D. Brazilin prevents against myocardial ischemia-reperfusion injury through the modulation of $\mathrm{Nrf2}$ via the PKC signaling pathway. Ann Transl Med 2021;9(4):312. doi: 10.21037/atm-204414
36. Jaiswal AK. Nrf2 signaling in coordinated activation of antioxidant gene expression. Free Radic Biol Med 2004;36:1199-207. 